\title{
Consideraciones analgésicas sobre el manejo del dolor agudo en el adulto mayor
}

\section{Considerations for acute pain analgesic management in the elderly}

\author{
Dr. Alfredo Covarrubias-Gómez,* Dr. Javier Alvarado-Pérez, ${ }^{\ddagger}$ \\ Luz A Templos-Esteban, ${ }^{\S}$ María López-Collada Estrada," \\ Centro Algia para la Educación en Salud
}

Citar como: Covarrubias-Gómez A, Alvarado-Pérez J, Templos-Esteban LA, López-Collada EM, Centro Algia para la Educación en Salud. Consideraciones analgésicas sobre el manejo del dolor agudo en el adulto mayor. Rev Mex Anest. 2021; 44 (1): 43-50. https://dx.doi.org/10.35366/97776

RESUMEN. En las últimas décadas se ha registrado un aumento dramático en la esperanza de vida. La principal morbilidad de este grupo poblacional son las enfermedades crónico-degenerativas, las cuales frecuentemente se acompañan de dolor. No es de extrañarse que cada vez más pacientes ancianos sean intervenidos quirúrgicamente; hasta $62 \%$ de ellos reportan dolor agudo postoperatorio (DAPO) severo. El envejecimiento se asocia a una respuesta reducida al estímulo doloroso, fenómeno conocido como presbialgesia, lo cual se traduce en menores requerimientos analgésicos. Cuando el paciente anciano cuenta con demencia u otras alteraciones de la cognición se deberán utilizar escalas conductuales. Existen diversas condiciones fisiológicas en el anciano que lo hacen propenso a acumulación de fármacos y retraso en su eliminación. Además, existe un riesgo aumentado de interacciones farmacológicas por polifarmacia, hechos que se deberán tener en cuenta al abordar clínicamente el dolor en el paciente geriátrico. Los antiinflamatorios no esteroideos (AINE) son considerados de riesgo en ancianos frágiles por aumentar el riesgo de sangrado, sin mencionar el potencial daño en pacientes nefrópatas o con trastornos de la coagulación. El paracetamol es un analgésico noble a nivel gástrico, renal y cardiovascular. Es considerado primera línea en osteoartrosis degenerativa. Se sugiere no sobrepasar dos gramos al día en ancianos frágiles. Al utilizar analgésicos opioides se sugiere iniciar con la mínima dosis analgésica y escalar lentamente en caso de que el alivio del dolor lo requiera, evitando así efectos adversos.

ABSTRACT. Over the last few decades the elderly population is growing by the second. Most of their illnesses are of the chronic degenerative type, many of which are very painful. Also, more and more elderly patients are requiring surgery for a number of reasons; $62 \%$ of these patients will experience severe postoperatory pain. Aging is associated with presbialgesia, which is a reduced response to a noxious stimule. When these patients have dementia or other cognitive impairment conductual scales such must be utilized. There elderly might be more sensitive to analgesic drugs due to various and unique physiological aspects. NSAIDs are considered high risk drugs in fragile elderly patients. Acetaminophen is a safe analgesic drug without many gastrointestinal, renal or cardiovascular secondary effects. Nevertheless, a fragile elderly patient should not receive more than 2 grams daily. When giving opioids, one should start with the minimum dose and titrate slowly when needed in order to avoid adverse effects.

\section{SOBRE EL CENTRO NACIONAL PARA LA EDUCACIÓN EN DOLOR (CNED) Y EL GRUPO DE APOYO AVANZADO EN DOLOR AGUDO (AADA)}

$\mathrm{L}$ a Asociación Mexicana para el Estudio del Dolor (AMETD) nace en 1981 como una organización enfocada a promover la educación y el estudio en materia del dolor. En 1993 dicha asociación es designada el capítulo mexicano de la Asociación Internacional para el Estudio del Dolor (IASP). La IASP es el asesor de la Organización Mundial de la Salud (OMS) sobre temas relacionados con el dolor y cuenta con más de 7 mil miembros en 133 países.

En 2012 el Grupo de Interés en Dolor Agudo de la AMETD inició un programa de educación en dolor agudo denominado «Apoyo Avanzado en Dolor Agudo» (AADA). El 5 de noviembre

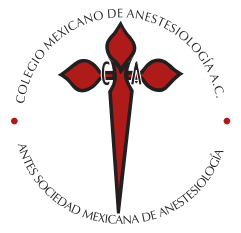

Palabras clave: Dolor, agudo, dolor postoperatorio, adulto mayor, geriatría.

Keywords: Pain, acute pain, postoperative pain, elderly, geriatrics.

* Departamento de Medicina del Dolor y Paliativa del Instituto Nacional de Ciencias Médicas y Nutrición «Salvador Zubirán».

Ciudad de México, México.

‡ División de Anestesiología y Clínica del Dolor del Instituto

Nacional de Rehabilitación. Ciudad de México, México.

$\S$ División de Cuidados Paliativos y Clínica del Dolor del Hospital General «Dr. Manuel Gea González». Ciudad de México, México.

" Departamento de Cuidados Paliativos. Centro Estatal de Cancerología de Chihuahua, Chihuahua.

Correspondencia: Dr. Alfredo Covarrubias-Gómez Departamento de Medicina del Dolor y Paliativa, Instituto Nacional de Ciencias Médicas y Nutrición «Salvador Zubirán». Vasco de Quiroga Núm. 15, Col. Sección XVI, 14000, Alcaldía Tlalpan, Ciudad de México. Teléfono: (+52-55) 54870900, ext. 5011

E-mail: alfredocov@yahoo.com cned.mexico@gmail.com

Recibido para publicación: 08-12-2016

Aceptado para publicación: 28-08-2019 
de 2015 este grupo se conforma en Mazatlán como el Centro Nacional para la Educación en Dolor (CNED) y está compuesto por médicos socios activos de la AMETD, de diferentes áreas, instituciones y regiones del país.

Como se mencionó los profesores del CNED son en su totalidad miembros activos de la AMETD. Sin embargo, no son voceros de la misma y tampoco representan los intereses de dicha agrupación gremial. Los miembros del CNED participan activamente en la revisión y búsqueda documental del material que se expone a continuación.

\section{LA IMPORTANCIA DE CONSIDERAR A LA POBLACIÓN GERIÁTRICA}

Los cambios en las pirámides poblacionales están redefiniendo las necesidades de la salud en la población moderna. En México, de 1990 al 2010, se identificó incremento en: la esperanza de vida (en 1990 de 71 años y en 2010 de 76 años), en la mediana de edad (en 1990 una mediana de 19 años y en 2010 de 26 años) y en la tasa de crecimiento de la población geriátrica (cercana a 4\% de 2000 a 2010) $)^{(1)}$.

En 2010 el país contaba con poco más de 10 millones de ancianos (uno de cada 10) y se estima que para 2029 se habrá duplicado el número de adultos mayores ${ }^{(1)}$. Estos datos sugieren que los mexicanos del futuro vivirán por más años.

La principal causa de morbilidad en los adultos mayores son las enfermedades crónico-degenerativas, éstas son responsables del incremento en la demanda de servicios de salud, y muchas de ellas se acompañan de dolor agudo o crónico ${ }^{(2,3)}$. Esto debe orientarnos hacia la previsión de servicio y atención para una población geriátrica creciente.

\section{FRECUENCIA DEL DOLOR AGUDO POSTOPERATORIO EN LA POBLACIÓN GERIÁTRICA}

En México 22.7\% de los internamientos hospitalarios se debieron a causas que requirieron una intervención quirúrgica $(794,500 \text { personas })^{(4)}$. Un estudio nacional demostró que $96.3 \%$ de los pacientes hospitalizados presentaron dolor ${ }^{(5)}$. Esta serie de datos pone de manifiesto que la presencia de dolor es una eventualidad frecuente en el ámbito hospitalario mexicano.

En el país la segunda causa de hospitalización en mayores de 60 años son las cirugías ${ }^{(3)}$; las cuales representaron $11.2 \%$ de los internamientos hospitalarios ${ }^{(5)}$. En un estudio nacional se identificó que $99 \%$ de los enfermos postquirúrgicos refirió dolor y $79 \%$ lo calificó como moderado a severo en intensidad ${ }^{(6)}$. El dolor agudo postoperatorio (DAPO) puede estar asociado con una enfermedad preexistente, al procedimiento quirúrgico o a una combinación de ambos ${ }^{(5)}$.
En la Unión Americana se ha documentado que 62\% de los adultos mayores que son intervenidos quirúrgicamente refieren DAPO severo en intensidad ${ }^{(7)}$. En México $23.6 \%$ de una muestra de 61 a 90 años de edad $(n=83)$ presentaron DAPO severo en intensidad durante las primeras 24 horas del postoperatorio (Garduño-López A, et al. Estudio aún no publicado). La presencia de DAPO en la población geriátrica incrementa la morbimortalidad asociada con la cirugía.

\section{DEFINICIÓN DE ANCIANIDAD Y CLASIFICACIÓN DEL ADULTO MAYOR}

Existen controversias sobre el rango de edad en el cual se considera a una persona adulto mayor (anciano, viejo o adulto en plenitud). Mientras que la Organización Mundial de la Salud (OMS) sitúa en ese rango a los mayores de 65 años, la Organización Panamericana de la Salud (OPS) considera en ese grupo a los mayores de 60 años. Otros autores consideran que diversas condiciones fisiológicas se modifican con la edad y han propuesto tres grupos en la categorización de los adultos mayores $(\text { Tabla } 1)^{(8)}$.

\section{EL IMPACTO DEL CONTROL DEL DOLOR POSTOPERATORIO EN EL DESENLACE}

En el adulto mayor se ha descrito la presencia de múltiples síndromes geriátricos como complicaciones postoperatorias, entre las que se encuentran:

- Delirium postoperatorio (incidencia de 15 a 53\%).

- Deterioro cognoscitivo y disfunción cognoscitiva postoperatoria (incidencia en cirugía no cardíaca de $25 \%$ en la primera semana posterior a la cirugía).

- Polifarmacia (incrementa el riesgo de discapacidad, hospitalización y muerte).

- Fragilidad en el adulto mayor postoperado (prevalencia de 4.1 a $50.3 \%)$.

- Abatimiento funcional o desacondicionamiento (presente en 30 a 60\% de los ancianos hospitalizados).

- Síndrome de caídas.

- Desnutrición.

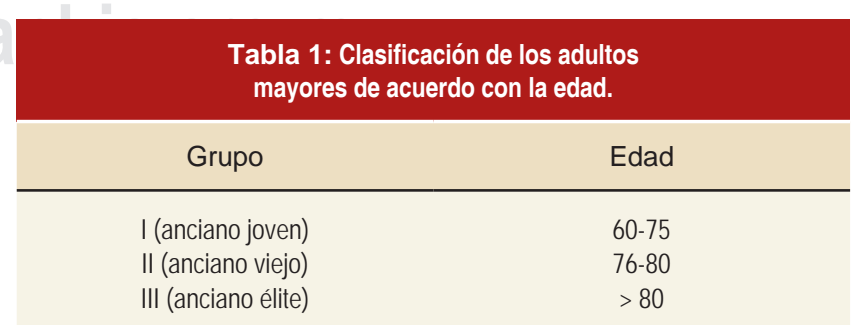


- Inmovilidad.

- Úlceras por presión.

- $\mathrm{DAPO}^{(9)}$.

El alivio del DAPO tiene un alto impacto en la calidad de vida de los enfermos. Se ha reportado que su manejo efectivo mejora las condiciones generales del paciente, facilita una recuperación más rápida y disminuye la estancia hospitalaria. Contrariamente su manejo ineficaz se ha asociado con la presencia de eventualidades potencialmente adversas (íleo, atelectasias, neumonía, tromboembolia, sangrado, alteraciones psicológicas, múltiples síndromes geriátricos, entre otras) (10). A pesar de que se ha fundamentado en la evidencia el beneficio de una analgesia óptima se estima que $64 \%$ de estos pacientes no reciben una terapéutica enfocada al alivio de su dolor, aun cuando éste es severo en intensidad ${ }^{(11)}$.

\section{EVALUACIÓN DEL DAPO Y OBJETIVOS DE LA ANALGESIA EN POBLACIÓN GERIÁTRICA}

\section{Objetivos de la analgesia postoperatoria en población geriátrica}

Los objetivos de la analgesia para el control del DAPO en general son: reducir la intensidad del dolor mediante una analgesia óptima; favorecer la normalización de la funcionalidad orgánica; disminuir la presencia de efectos adversos; prevenir y reducir la presencia de complicaciones atribuibles a la cirugía o a la descompensación de enfermedades previas; favorecer mejores condiciones asociadas con la vida; impactar favorablemente en la satisfacción del enfermo con la atención médica recibida, entre otras ${ }^{(10)}$.

La evaluación clínica del dolor en el anciano tiene objetivos similares a los que se esperan en poblaciones de adultos jóvenes; sin embargo, las características especiales de la población geriátrica hacen que tal evaluación sea un reto para el clínico ${ }^{(11)}$. Con frecuencia los adultos mayores son excluidos de los ensayos clínicos sobre evaluación y tratamiento del dolor por la presencia de alteraciones cognitivas o sensoriales. Estas eventualidades conducen a que en esta población se infravalore la intensidad de su dolor y a que se proporcione una analgesia deficiente ${ }^{(12)}$.

Se ha demostrado que los beneficios de una analgesia óptima son idénticos a cualquier edad ${ }^{(12)}$. Por lo anterior, independientemente del grado de dificultad que presente la evaluación del DAPO en el paciente geriátrico, el objetivo final es proporcionar un exitoso manejo del dolor ${ }^{(11)}$.

\section{Retos para la evaluación del dolor en el adulto mayor}

El adulto mayor presenta algunas diferencias para reportar el dolor y éstas pueden ser atribuidas a diferentes factores, entre los que destacan: factores biológicos, culturales, étni- cos, religiosos, alteraciones cognitivas y contextos sociales u organizacionales ${ }^{(12)}$.

Entre estos factores destacamos los siguientes puntos:

- Los cambios neurobiológicos relacionados con el dolor y asociados al proceso de envejecimiento pueden tener un impacto directo sobre la farmacología de los analgésicos y la fisiopatología de la nocicepción ${ }^{(13)}$.

- Las alteraciones cognitivas, el aislamiento social y las limitaciones físicas son otro factor frecuentemente ignorado y que impacta sobre la accesibilidad al tratamiento contribuyendo al manejo inapropiado del dolor ${ }^{(13)}$.

- La presencia de comorbilidades incrementa el riesgo de efectos adversos o complicaciones asociadas con la analgesia ${ }^{(12)}$.

- Los grupos médicos con frecuencia carecen del entrenamiento suficiente sobre el manejo de opioides en población geriátrica, lo cual limita su empleo por miedo y opioidefobia ${ }^{(12)}$.

Aunado a estas consideraciones se debe tomar en cuenta la presencia de barreras conceptuales que impactan en el dolor del adulto mayor, éstas incluyen la creencia de que los viejos experimentan menos dolor que otros grupos poblacionales. No es para sorprenderse que los propios pacientes geriátricos crean que el dolor es algo que debe soportarse, que los analgésicos causen adicciones, que el quejarse del dolor es signo de debilidad y que el dolor es una parte inevitable del envejecimiento $^{(12)}$.

\section{La percepción del dolor por el paciente geriátrico}

El envejecimiento se encuentra asociado con un cierto grado de presbialgesia (respuesta reducida a un estímulo normalmente doloroso). Este fenómeno es ejemplificado en modelos de dolor visceral (infartos miocárdicos silentes, apendicitis indolora del anciano, entre otras). Estas características identificadas en el adulto mayor sugieren que las sensaciones nociceptivas agudas provenientes de estructuras profundas se encuentran reducidas ${ }^{(12)}$.

El hecho de que exista hipoalgesia visceral no significa que exista hipoalgesia periférica. Se ha demostrado que a nivel periférico los estímulos dolorosos agudos (térmicos) presentan el mismo reporte en intensidad en jóvenes y ancianos ${ }^{(12)}$.

Existe la posibilidad de que la modulación del dolor presente modificaciones asociadas al envejecimiento que condicione una percepción del dolor diferente a la del paciente joven (la despolarización periférica es similar en jóvenes y viejos, la sumación espacial y la modulación cortical se encuentran disminuidas en los adultos mayores, existen diversas axonopatías asociadas con comorbilidades en el paciente geriátrico, etcétera).

La suposición de presbialgesia en el anciano jamás debe ser motivo para limitar el manejo del dolor en el enfermo geriátrico $^{(12)}$. 


\section{El autorreporte del dolor percibido}

El autorreporte, hasta el momento, continúa siendo la forma más confiable para evaluar el dolor. La evaluación por el personal de salud o los cuidadores del enfermo con frecuencia lo infraestiman o sobrestiman. En el paciente geriátrico el interrogatorio para obtener el autorreporte del dolor es el primer paso en su evaluación ${ }^{(12,14)}$. No obstante, aunque el dolor es una experiencia subjetiva, las escalas uni- y multidimensionales siguen siendo útiles y confiables si son aplicadas apropiadamente ${ }^{(12)}$.

\section{Evaluación cualitativa y cuantitativa del dolor en geriatría}

La evaluación cualitativa y cuantitativa del dolor constituye la mejor herramienta para la evaluación del dolor por el paciente anciano $^{(12)}$. La evaluación de la intensidad del DAPO (cuantitativa) ha utilizado diversas escalas (Figura 1), entre las que se encuentran:

- La escala visual análoga (EVA) en población geriátrica. Este instrumento presenta cierto grado de complejidad identificándose que a mayor edad existe más riesgo de confusión al utilizar esta escala ${ }^{(15)}$. Hasta 30\% de los adultos mayores sin alteraciones cognitivas presentan problemas con esta escala ${ }^{(14)}$.
- La escala verbal numérica o escala numérica análoga (ENA). Aunque ha sido validada en población geriátrica presenta las mismas limitaciones que la $\mathrm{EVA}^{(16)}$.

- La escala verbal análoga (EVERA), desarrollada por Melzack y originalmente con seis categorías, es considerada el instrumento de elección para la evaluación cuantitativa del dolor en población geriátrica ${ }^{(17)}$.

- La escala de caras modificada (ECM). Esta escala ha sido la más preferida y la más confiable en los pacientes geriá$\operatorname{tricos}^{(18,19)}$.

Existen otras escalas cuantitativas para evaluar la intensidad del dolor en el enfermo geriátrico, todas ellas incluyen entre cinco y seis ítems que se evalúan con categorías similares a las usadas en la EVERA, entre estas se encuentran: FPS (functional pain scale o escala funcional de dolor), DVS (descriptive verbal scale o escala verbal descriptiva), PPI (present pain intensity o intensidad presente del dolor), GPA (global pain assessment o evaluación global del dolor), entre otras ${ }^{(14,20)}$.

La evaluación cualitativa del dolor incluye la identificación de diversas variables entre las que se encuentran: localización o ubicación del dolor, extensión y dinámica del dolor, características del dolor (cuestionario McGill), factores agravantes o desencadenantes, funcionalidad (evaluación de Katz sobre actividades diarias, evaluación de Lawton para actividades instrumentadas, escala de Barthel), alteraciones

Escala para evaluar la intensidad y severidad del dolor

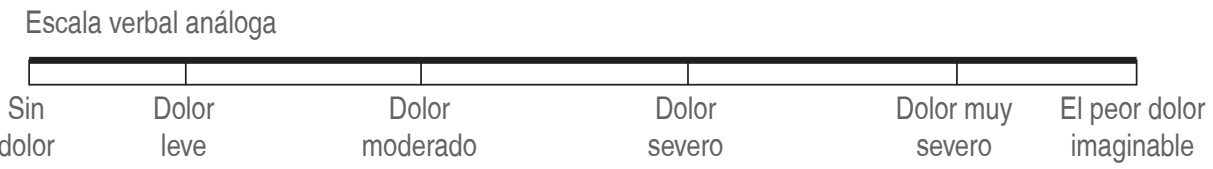
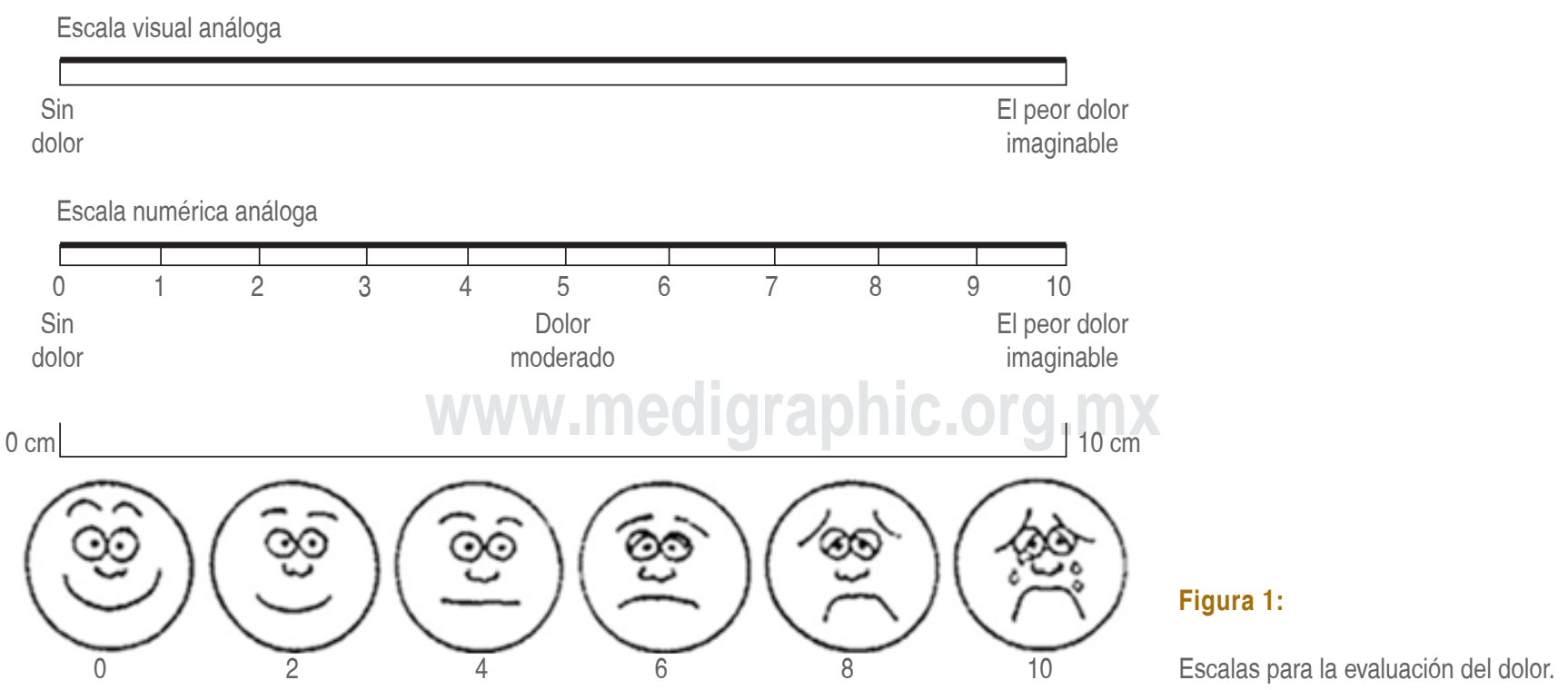
psicoafectivas relacionadas con la presencia de dolor (escala geriátrica de depresión), alteraciones cognitivas (DSM-V) y alteraciones del apetito o del dormir, además la reevaluación frecuente es esencial.

Dos instrumentos que evalúan el dolor en forma multidimensional han sido validados en población geriátrica, éstos son: el inventario breve de dolor y el índice de discapacidad por dolor ${ }^{(14,20)}$.

\section{Evaluación del dolor en el enfermo geriátrico con demencia}

En los adultos mayores con alteraciones cognitivas la evaluación del dolor presenta un reto para el clínico. Esta población en particular se encuentra en riesgo de recibir un esquema analgésico deficiente dadas las dificultades que pueden estar presentes para expresar su dolor ${ }^{(21)}$.

Es conveniente que el clínico evalué, aun en el paciente con demencia: presencia o ausencia de dolor o incomodidad, intensidad del dolor, su localización, frecuencia e impacto en sus actividades diarias. Los pacientes con una alteración cognitiva inicial o intermedia son capaces de expresar su dolor en mayor o menor grado. Los pacientes con demencia severa, déficit cognitivo agudo severo o incapacidad para la comunicación por deterioro funcional son evaluados de forma diferente (Tabla 2)(12,20).

Existen otras escalas como el PAINAD (pain assessment in advanced dementia o evaluación del dolor en demencia avanzada), PACSLAC (pain checklist for seniors with limitations to communicate o listado de dolor para adultos mayores con limitaciones para la comunicación), DOLOPLUS-2 (instrumento francés traducido y validado en cinco idiomas diferentes incluido el español), ECPA-2 (elderly pain caring assessment-2 o evaluación del dolor en el adulto mayor por el cuidador), Abbey Pain Scale, Mahoney Pain Scale, entre otras, las cuales parecen prometedoras para la evaluación de estos pacientes ${ }^{(12,14,20)}$.

\section{CAMBIOS DURANTE EL ENVEJECIMIENTO QUE MODIFICAN LOS PROCESOS FARMACOLÓGICOS}

Los cambios en los procesos farmacológicos durante el envejecimiento se enlistan en la Tabla 3.

La absorción de los fármacos se realiza principalmente por difusión pasiva y esto ocurre de manera predominante en el tracto gastrointestinal. En el adulto mayor este proceso no sufre modificaciones significativas asociadas con la edad. Sin embargo, es conveniente identificar que en este grupo poblacional la motilidad intestinal disminuye y el $\mathrm{pH}$ gástrico se incrementa. Teóricamente las modificaciones del pH modificarían la absorción de los medicamentos y el hecho de que se incremente el tiempo de absorción explica el que no se modifique este proceso a pesar de estos cambios ${ }^{(22,23)}$.
Tabla 2: Evaluación del dolor en el enfermo con déficit

cognitivo agudo severo o demencia severa o limitación

para la comunicación asociada a deterioro cognitivo sugerida por el American Geriatrics Society.

Expresión facial

Vocalización o verbalización

Movimientos corporales

Cambios en su relación interpersonal con cuidadores y familiares

Cambios en sus actividades o rutinas cotidianas

Disminución de la habilidad funcional/aislamiento

Fluctuación del estado cognitivo/estado hipoactivo o hiperactivo

Otras condiciones fisiológicas presentes en el adulto mayor y que se han asociado con la acumulación de fármacos, retraso en la eliminación de los mismos e incremento en la ocurrencia de interacciones farmacológicas son las siguientes ${ }^{(22,23)}$ :

- El incremento en la grasa corporal, la disminución de la masa muscular y la reducción del agua corporal afectan al volumen de distribución. Por ello, los fármacos que son liposolubles permanecen más tiempo en el organismo.

- Disminución de $12 \%$ en la albúmina sérica. La hipoproteinemia por hipoalbuminemia condiciona que disminuya la unión proteica a fármacos ácidos (levotiroxina, digoxina, warfarina, fenitoína).

- En la hipoproteinemia por déficit de alfa-1 glicoproteína disminuye la unión proteica a fármacos básicos (lidocaína, propanolol).

- Disminuye de la función hepática por un decremento del flujo sanguíneo hepático (25 a 47\%) y disminuye la oxidación microlisosomal.

- Cambios metabólicos asociados al proceso de envejecimiento. Disminuye el metabolismo de fase I por cambios en el CYP450 (cambio de 3A4 a 2D6), y de fase II por decremento en la actividad enzimática de conjugación.

- El flujo renal disminuye 1\% por año después de los 50 años, y también se ve una baja en la depuración de creatinina (depuración de creatinina $\leq 30$ ). El impacto de esta variable fisiológica se observa en fármacos con más de $60 \%$ de eliminación renal.

- Se debe considerar la «sensibilidad a fármacos»o «sensibilización farmacológica». Este fenómeno se caracteriza por una respuesta intrínseca anormal a una dosis estándar y es debido a cambios farmacocinéticos que afectan a la farmacodinamia. Esto favorece que se presente una mayor sensibilidad a los efectos sedantes de las benzodiacepinas, warfarina y opioides, lo cual disminuye los requerimientos de estos fármacos, y que por el contrario exista una menor sensibilidad a los efectos de fármacos beta-adrenérgicos, incrementado sus requerimientos. 
- En el anciano las modificaciones del sistema nervioso autónomo (parasimpático) se han relacionado con el efecto de diversos fármacos y que los sistemas homeostáticos se encuentran alterados.

\section{ABORDAJE DEL DAPO EN EL ADULTO MAYOR}

El hecho de que las terapias convencionales para el alivio del dolor sean verificadas y evidenciadas (aun en forma metaanalítica) en poblaciones jóvenes no significa que puedan ser aplicadas al paciente geriátrico en forma automática y sin considerar todos aquellos factores característicos del proceso de envejecimiento ${ }^{(13)}$.

Se ha documentado que el dolor agudo postoperatorio del enfermo geriátrico es infratratado ${ }^{(7,24)}$. El manejo inapropiado del dolor puede producir síndromes dolorosos crónicos difíciles de tratar, agravar algunos síndromes e impactar negativamente en las condiciones asociadas con la vida del enfermo anciano ${ }^{(12)}$.

En el adulto mayor la presencia de eventos adversos asociados con la farmacoterapia es más frecuente que en poblaciones más jóvenes, y esto ocurre a pesar del empleo de dosis en ocasiones infraterapéuticas. Diversos elementos pueden contribuir a este fenómeno, entre los que se encuentran: la polifarmacia y cambios metabólicos y de eliminación relacionados al envejecimiento ${ }^{(14)}$. Esta serie de elementos aunados a las potenciales interacciones farmacológicas pueden condicionar que los clínicos en aras de ser más cautelosos limiten la analgesia en sus enfermos.

Durante la última década se han descrito múltiples guías clínicas para el manejo del dolor agudo; sin embargo, no se focalizan en la población geriátrica. Algunas publicaciones consensuadas sugieren que la utilización de «la escalera analgésica» de la Organización Mundial de la Salud sea tomada en consideración para el manejo de esta población; entre éstas, se encuentran las recomendaciones de la Sociedad Geriátrica Americana ${ }^{(14)}$. Este abordaje no siempre es de utilidad, ya que el enfermo geriátrico puede presentar diversas comorbilidades que orientan hacia un manejo más acucioso ${ }^{(10)}$. Un ejemplo sería el caso de un enfermo geriátrico portador de neuropatía diabética dolorosa, osteoartritis en múltiples articulaciones, consumidor crónico de opioides, sometido a artroplastía de rodilla y que presenta insuficiencia aguda prerrenal en el postoperatorio: A. Considerando la escalera, la utilización de antiinflamatorios no esteroideos no es recomendable en el enfermo con enfermedad renal. B. Este paciente requiere una dosis de opioide mayor al consumo basal de los que ya consume. C. Las técnicas de anestesia-analgesia neuroaxial o regional para extremidades inferiores no modificarán la presencia de dolor en las extremidades superiores o en hombros. D. La neuromodulación de la neuropatía periférica con antidepresivos pudiera impactar en complicaciones postoperatorias (como la presencia de sangrado).

\section{CONSIDERACIONES ASOCIADAS CON LA FARMACOTERAPIA DEL DAPO}

\section{Analgésicos no opioides}

Los antiinflamatorios no esteroideos (AINE) ya sea «selectivos» o «no selectivos» son considerados un riesgo en la su-

\begin{tabular}{|c|c|c|c|}
\hline Principio farmacológico & & Cambio fisiológico & Relevancia clínica \\
\hline \multirow[t]{15}{*}{ Farmacocinética } & Absorción & Disminuye la superficie de absorción & \\
\hline & & Disminuye el flujo esplácnico & \\
\hline & & Incrementa el pH gástrico & \\
\hline & & Alteración en la movilidad intestinal & Existen pocos cambios en la absorción del paciente anciano \\
\hline & Distribución & Disminuye el agua corporal total & Incrementa la concentración de los fármacos que se distribuyen \\
\hline & & Disminuye la masa muscular & en los líquidos corporales \\
\hline & & Incrementa la masa magra & El caso de los fármacos liposolubles \\
\hline & & Disminuye la albúmina sérica & Incremento en la fracción libre en el plasma del fármaco \\
\hline & & Alteración en la unión a proteínas & \\
\hline & Metabolismo & Reducción de la masa hepática & Disminuye el metabolismo de primer paso \\
\hline & & Reducción del flujo sanguíneo hepático & Disminuye la tasa de biotransformación de algunos fármacos \\
\hline & & Disminución del metabolismo de fase I & Incrementa el riesgo de interacciones farmacológicas \\
\hline & Eliminación & Disminución del flujo sanguíneo renal & La disminución de la eliminación renal de los fármacos y sus \\
\hline & & Disminuye la tasa de filtración glomerular & metabolitos depende del grado de funcionalidad renal \\
\hline & & Disminuye la función de secreción tubular & \\
\hline \multirow[t]{2}{*}{ Farmacodinamia } & & Alteración en el número de receptores & El anciano es más/menos sensible a diversos fármacos \\
\hline & & Alteración en la afinidad de receptores & \\
\hline
\end{tabular}


pervivencia del anciano «frágil» ${ }^{(25)}$. El manejo del DAPO con estos medicamentos puede favorecer el «riesgo de sangrado» tras su administración. En mayores de 60 años el «riesgo de sangrado» se incrementa a $34 \%$ y en aquellos ancianos con historia de sangrado previo aumenta a $9 \%(26)$.

Ante este potencial riesgo el empleo de combinaciones de AINE con misoprostol, antiácidos, antagonistas de los receptores $\mathrm{H} 2$ o inhibidores de la bomba de protones puede ser útil en la población geriátrica. No obstante, a lo ya mencionado y derivado de la polifarmacia, es conveniente señalar que los protectores gastrointestinales pueden alterar la función renal y promover la presencia de interacciones farmacológicas ${ }^{(25)}$.

El paracetamol tiene un mecanismo analgésico diferente al de los AINE. Este fármaco proporciona una analgesia sin riesgo de gastrolesividad, nefrolesividad, cardiolesividad, anormalidades en la coagulación o alteraciones en la cicatrización y consolidación ósea que se presentan con los AINE(26). Dada su seguridad, este fármaco ha sido recomendado como primera línea de manejo para el tratamiento del dolor asociado con osteoartritis ${ }^{(25)}$. A pesar de la benevolencia aparente del paracetamol se deben tener en consideración algunos aspectos. Su aclaramiento disminuye en el paciente anciano especialmente en el enfermo «frágil» ${ }^{(25)}$. Este factor sugiere que en esta población las dosis administradas deben ser menores a las recomendadas y algunos autores proponen no exceder los 2 gramos al día ${ }^{(27)}$.

Por otro lado, en el enfermo que utiliza anticoagulantes de tipo warfarínico existe una interacción con paracetamol que favorece el «riesgo de sangrado» ${ }^{(28)}$. Esta potencial interacción afecta las concentraciones plasmáticas de los anticoagulantes ${ }^{(27)}$; por lo que en el enfermo geriátrico que lo consume crónicamente se debe tener una monitorización cercana del índice internacional normalizado (INR).

Otras eventualidades asociadas al paracetamol y que impactan a la población geriátrica son: la precipitación de cuadros asmáticos ${ }^{(29)}$, los cuales pueden ser potencialmente lesivos en el anciano bronquítico crónico; interacciones farmacológicas con algunos antibióticos (flucloxacilina) que pueden manifestarse como alteraciones de tipo ácido-base ${ }^{(30)}$; precipitación de cuadros hipotensivos en personas «frágiles» ${ }^{(31)}$, interacciones con algunas estatinas (simvastatina) que pueden favorecer la hepatotoxicidad( ${ }^{(32)}$, entre otras.

\section{Analgésicos opioides}

El manejo del DAPO en el enfermo geriátrico con opioides sugiere una evaluación cercana debido a sus potenciales efectos indeseables. Los problemas asociados con su empleo se ven favorecidos por los cambios en el metabolismo asociados al proceso de envejecimiento y que traen consigo acumulación de los metabolitos activos de algunos opioides ${ }^{(25)}$.

Entre las manifestaciones por la inversión en las isoformas del CYP-450 y la disminución en las enzimas del sistema microlisosomal se encuentra un incremento en la prevalencia de caídas y fracturas, alteraciones cognitivas incluyendo delirium, estreñimiento que requiere medidas invasivas asociadas con la disfunción intestinal por empleo de opioides, depresión respiratoria, entre otros ${ }^{(25,27)}$.

Cuando se emplean opioides endovenosos se sugiere «iniciar con la mínima dosis analgésica efectiva y realizar escaladas lentas en caso de que el alivio del dolor lo requiera» esto con la finalidad de que no se suspenda la terapéutica por considerarla «ineficaz» o por la presencia de efectos indeseables ${ }^{(27)}$.

En el postoperatorio del adulto mayor que recibe opioides se ha recomendado:

- Disminuir las dosis de opioides debido a los cambios en el metabolismo.

- Promover la utilización de opioides bajo una vigilancia estricta y ajustes cautelosos.

- Facilitar la analgesia multimodal a fin de limitar las dosificaciones elevadas de opioides.

- Suministrar en aras de aliviar el dolor de moderado a severo en intensidad de forma eficiente.

- Individualizar los casos y monitorizar las funciones renal y hepática tomando en consideración que ambas se encuentran afectadas en el enfermo anciano y en especial en el «frágil».

- La «analgesia controlada por el paciente» no debe limitarse en el paciente geriátrico (considerar estado cognitivo, incrementar el intervalo entre rescates, evaluar el momento ideal de la reducción o destete de opioides).

- Considerar las potenciales interacciones de los opioides con doble mecanismo de acción (antidepresivos, anticoagulantes, antibióticos).

- Monitorizar puntualmente los efectos adversos (neurotoxicidad, prurito, constipación, mareo, ataxia, caídas, náusea, mareo, vómito, delirium, sedación, afectación en la memoria, hipoxia, depresión respiratoria, entre otros) (33-35).

\section{CONCLUSIONES}

En los pacientes geriátricos el manejo del dolor mejora sus condiciones de vida, por ello, debemos proporcionarles este beneficio. Asimismo, los cambios propios del envejecimiento los hacen más susceptibles al efecto de los fármacos. De tal forma, que el abordaje farmacológico racional debe considerar los aspectos fisiológicos y emocionales que comúnmente se presentan en este grupo. Finalmente, el adulto mayor debe abordarse de forma integral y multidisciplinaria. Como en otros casos, es necesario generar la evidencia suficiente que norme nuestras conductas respecto al impacto que los fármacos tienen en nuestros pacientes. 
Conflicto de intereses: Los autores del presente documento no declaramos conflicto alguno de intereses. Hemos dado pláticas para la industria farmacéutica sin tener un contra- to laboral. No representamos los intereses de terceros. No recomendamos indicaciones fuera de la información para prescribir (IPP) de los medicamentos.

\section{REFERENCIAS}

1. Instituto Nacional de Estadística y Geografía (INEGI). Del portal en Internet sección Estadística y tema Población. [Fecha de consulta: agosto de 2012] Disponible en: http://www.inegi.org.mx/Sistemas/temasV2/ Default.aspx?s=est\&c $=17484$

2. Borges-Yáñez SA, Gómez-Dantés H. Uso de los servicios de salud por la población de 60 años y más en México. Salud Publica Mex. 1998;40:13-23.

3. Olaiz-Fernández G, Rivera-Dommarco J, Shamah-Levy T, Rojas R, Villalpando-Hernández S, Hernández-Ávila M, et al. Encuesta Nacional de Salud y Nutrición 2006. Cuernavaca, México: Instituto Nacional de Salud Pública; 2006.

4. Valdespino JL, Olaiz G, López-Barajas MP, Mendoza L, Palma O, Velázquez O, et al. Encuesta Nacional de Salud 2000. Tomo I. Vivienda, población y utilización de servicios de salud. Cuernavaca, Morelos, México: Instituto Nacional de Salud Pública; 2003.

5. Guevara-López U, Covarrubias-Gómez A, Hernández-Ortiz A. Desarrollo de los parámetros de práctica para el manejo del dolor agudo. Rev Mex Anest. 2004;27:200-204.

6. Hernández-Hernández L. Experiencia mexicana de la Unidad de Dolor Agudo: Instituto Mexicano del Seguro Social (IMSS). Rev Mex Anest. 2013;36:S176-S178.

7. Sauaia A, Min SJ, Leber C, Erbacher K, Abrams F, Fink R. Postoperative pain management in elderly patients: correlation between adherence to treatment guidelines and patient satisfaction. J Am Geriatr Soc. 2005;53:274-282.

8. Leininger SM. Managing pain in the older adult patient. Topics Emerg Med. 2002;24:10-18.

9. Instituto Mexicano del Seguro Social. Guía de Práctica Clínica: Manejo de síndromes geriátricos asociados a complicaciones postoperatorias. Catálogo maestro de GPC: IMSS-612-13.

10. Guevara-López U, Covarrubias-Gómez A, Delille-Fuentes R, HernándezOrtiz A, Carrillo-Esper R, Moyao-García D. Parámetros de práctica para el manejo del dolor agudo perioperatorio. Cir Cir. 2005;73:223-232.

11. Bruckenthal P. Assessment of pain in the elderly adult. Clin Geriatr Med. 2008;24:213-236.

12. Catananti C, Gambassi G. Pain assessment in the elderly. Surg Oncol. 2010;19:140-148.

13. McCleane G. Pharmacological pain management in the elderly patient. Clin Interv Aging. 2007;2:637-643.

14. Andrade DC, Faria JW, Caramelli P, Alvarenga L, Galhardoni R, Siqueira SR, et al. The assessment and management of pain in the demented and non-demented elderly patient. Arq Neuropsiquiatr. 2011;69:387-394.

15. Kremer E, Atkinson JH, Ignelzi RJ. Measurement of pain: patient preference does not confound pain measurement. Pain. 1981;10:241-248.

16. Wynne CF, Ling SM, Remsburg R. Comparison of pain assessment instruments in cognitively intact and cognitively impaired nursing home residents. Geriatr Nurs. 2000;21:20-23.

17. Herr KA, Spratt K, Mobily PR, Richardson G. Pain intensity assessment in older adults: use of experimental pain to compare psychometric properties and usability of selected pain scales with younger adults. Clin J Pain. 2004;20:207-219.
18. Stuppy DJ. The faces pain scale: reliability and validity with mature adults. Appl Nurs Res. 1998;11:84-89.

19. Taylor LJ, Herr K. Pain intensity assessment: a comparison of selected pain intensity scales for use in cognitively intact and cognitively impaired African American older adults. Pain Manag Nurs. 2003;4:87-95.

20. Hadjistavropoulos T, Herr K, Prkachin KM, Craig KD, Gibson SJ, Lukas A, et al. Pain assessment in elderly adults with dementia. Lancet Neurol. 2014;13:1216-1227.

21. Fremptom M. Experience assessment and management of pain in people with dementia. Age Ageing. 2003;32:248-251.

22. Bressler R, Bahl JJ. Principles of drug therapy for the elderly patient. Mayo Clin Proc. 2003;78:1564-1577.

23. Power I. Recent advances in postoperative pain therapy. Br J Anaesth. 2005;95:43-51.

24. Poisson-Salomon AS, De Chambine S, Lory C. Patient-related factors and professional practices associated with postoperative pain. Rev Epidemiol Sante Publique. 2005;53:1S47-1S56.

25. McLachlan A, Bath S, Naganathan V, Hilmer SN, Le Couteur DG, Gibson SJ, et al. Clinical pharmacology of analgesic medicines in older people: impact of frailty and cognitive impairment. Br J Clin Pharmacol. 2011;71:351-364.

26. Covarrubias-Gómez A, González-García JL, Betancourt-Sandoval JA, Mendoza-Reyes J. El dolor agudo perioperatorio y el paracetamol: una visión basada en la evidencia. Rev Mex Anest. 2013;36:47-55.

27. Covarrubias-Gómez A. Manejo del dolor en el paciente anciano. Rev Mex Anestesiol. 2006;29:S207-S209.

28. Mahé I, Bertrand N, Drouet L, Simoneau G, Mazoyer E, Bal dit Sollier C, et al. Paracetamol: a hemorrhagic risk factor in patients on warfarin. Br J Clin Pharmacol. 2005;59:371-374.

29. Dharmage SC, Allen KJ. Does regular paracetamol ingestion increase the risk of developing asthma? Clin Exp Allergy. 2011;41:459-460.

30. Jessurun N, van Marum R, Hermens W, van Puijenbroek E. Advanced age and female sex as risk factors for high anion gap metabolic acidosis after a drug interaction between paracetamol and flucloxacillin: a case series. J Am Geriatr Soc. 2016;64:e90-e93.

31. Krajčová A, Matoušek V, Duška F. Mechanism of paracetamol-induced hypotension in critically ill patients: a prospective observational crossover study. Aust Crit Care. 2013;26:136-141.

32. Gumbrevičius G, Sveikata A, Sveikatienė R, Stankevičius E. Paracetamol and simvastatin: a potential interaction resulting in hepatotoxicity. Medicina (Kaunas). 2012;48:379-381.

33. Aubrun F, French Society of Anesthesia and Resuscitation. Postoperative morphine administration in the elderly patient. Ann Fr Anesth Reanim. 2009;28:e39-e41.

34. Fine PG. Pharmacological management of persistent pain in older patients. Clin J Pain. 2004;20:220-226.

35. Gagliese L, Jackson M, Ritvo P, Wowk A, Katz J. Age is not an impediment to effective use of patient-controlled analgesia by surgical patients. Anesthesiology. 2000;93:601-610. 